\title{
OXIDATIVE STRESS, TELOMERE LENGTH, AND FRAILTY IN AN OLD AGE POPULATION
}

\author{
José D. Martínez-Ezquerro ${ }^{1,2}$, Aleida Rodríguez-Castañeda ${ }^{3}$, Mauricio Ortiz-Ramírez ${ }^{1}$, \\ Sergio Sánchez-García ${ }^{4}$, Haydee Rosas-Vargas ${ }^{1}$, Rosalinda Sánchez-Arenas ${ }^{4}$, \\ and Paola García-de la TORRE ${ }^{3}$
}

${ }^{1}$ Human Genetics Medical Research Unit, Pediatrics Hospital, Centro Médico Nacional Siglo XXI, Instituto Mexicáno del Seguro Social, Mexico City; ${ }^{2}$ Biological Sciences Graduate Program, Universidad Nacional Autónoma de Méxicoo, Mexico City; ${ }^{3}$ Neurological Diseases Medical Research Unit, Specialties Hospital, and "Epidemiology and Health" Services Research Unit, Aging Area, Centro Médico Nacional Siglo XXI, Instituto Mexicano del Seguro Social, Mexico City, Mexico

\begin{abstract}
\end{abstract}
Background: A global aging population requires focusing on the risk factors for unhealthy aging, preventive medicine, and chronic disease management. The identification of adverse health outcomes in older adults has been addressed by the characterization of frailty as a biological syndrome. In this field, oxidative stress and telomere length have been suggested as biomarkers of aging. Objective: The objective of the study was to study the association of oxidative stress, telomere length, $^{2}$ and frailty in an old age population. Methods: We conducted a cross-sectional study based on 2015 data from 202 members of a cohort of older adults ( $n=202$; F/M gender ratio: 133/69; mean age: $69.89 \pm 7.39$ years). Reactive oxygen species were measured by dichlorofluorescein diacetate and lipid peroxidation by malondialdehyde. Telomere length was determined using quantitative polymerase chain reaction with SYBR Green Master Mix. Results: Statistical analysis showed an assốciation between telomere length and frailty but no association between oxidative stress and telomere length or frailty. Conckusions: Telomere length could eventually be used as a marker to differentiate between healthy and unhealthy aging as expressed by frailty phenotype; oxidative stress seemed merely a biological process of aging. (REV INVEST CLIN. 2019;71:393-401)

Key words: Healthy aging. Biomarkers. Homeostasis. Frailty.

\section{INTRODUCTION}

A global aging population has motivated researchers to focus on risk factors, preventive medicine, and chronic diseases in preparation for the coming health issues that this phenomenon will cause. The international economy and society itself will suffer from the worldwide changes in population age distribution. Between the years 2015 and 2050, the proportion of the world's population older than 60 years will increase from 900 million to 2000 million, $\_$a rise from $12 \%$ to $22 \%{ }^{1}$. In 2015 , Mexico had one older adult ( $\geq 60$ years of age) for every 10 younguadults (15 years old), a figure that is expected to double
*Corresponding author:

Paola García-de la Torre

E-mail: pgarciatorre@gmail.com
Received for publication: 28-05-2019

Approved for publication: 30-08-2019

DOI: $10.24875 / R I C .19003116$ 
by $2050^{2}$. At present, Mexico City is the entity in Mexico with the highest index of aging, 59.1 in 2014, and a projection of 108.7 by $2030^{3}$. Due to this global demographic transition, successful aging (prevention of disease/disability, high physical and cognitive function, and sustained engagement in social activities) has become a priority ${ }^{4}$.

The concept of aging suggests the emergence and accumulation of chronic conditions such as heart disease, diabetes mellitus (DM), cancer, and cerebrovascular diseases, which are also the main causes of death at this stage of life. Hence, the identification of adverse health outcomes in older adults has been addressed by the characterization of frailty as a biological syndrome. Frailty has been described as the loss of physiologic reserve and resistance to stressors that involves multiple physical, mental, and emotional deficits ${ }^{5}$. Frailty increases vulnerability to dependence and the risk of adverse events such as functional decline, falls, hospitalization, institutionalization, and mortality ${ }^{5,6}$. In Mexico City, a prevalence of frailty of $20.6 \%$ among older adults has been reported ${ }^{7}$, an intermediate level when compared with global reports from $4 \%$ to $59.1 \%^{8}$. Hence, the search for biomarkers that can help define frailty at a biological level could be used to improve the diagnosis of frailty, to identify pre-frail patients at an earlier stage, and to evaluate the biological outcome when frailty is modified by factors such as healthy eating or physical exercise. On the matter, telomere length, which has been associated with lifespan ${ }^{9}$, has had a controversial role in frailty description. Although no correlation was found for Chinese ${ }^{10}$ and German ${ }^{11}$ populations, we recently observed shorter telomeres associated with frailty in a Mexican population ${ }^{12}$.

Telomeres are protective and regulatory structures of the genome; their shortening is associated with chronic degenerative diseases such as $\mathrm{DM}^{13}$, obesity, and hypertension ${ }^{14,15}$ and has been associated with lifespan ${ }^{9}$. Lifespan depends on many internal and external factors such as aging, pollution, quality of life, and education, among others. Consequently, telomere shortening and frailty would seem to be intimately related. Most of the associations with telomere length mentioned above involve the production of oxidative stress that can affect DNA in a direct form. Oxidative stress has been associated with chronic damage in several conditions, including DM and hypertension9,16-18. At a molecular level, $0 x i d a-$ tive stress is capable of altering DNA and has been linked to telomere length reduction ${ }^{19}$. To determine the possible association between reactive gxygen species (ROS), telomere length, and frailty, we evaluated the effect of oxidative stress on tetomere length as a first approach to explain telomerêlength shortening in frail patients. Then, we assessed both telomere length and oxidative stress, as biomarkers of frailty.

\section{METHODS}

\section{Participants}

This is a cross-sectional study based on 2015 data from the "Cohort of Obesity, Sarcopenia, and Frailty of Older Mexican Adults" (COSFOMA), a study involving 1252 adults $\geq 60$ years of age affiliated to the Mexican Social Security Institute (IMSS), and who were residents of Mexico City ${ }^{7}$. The IMSS is part of the social protection system in health in Mexico, which provides services to salaried workers and their families, including medical care and economic bènefits such as disability pensions or paid retirement. 回Mexico City, IMSS has 48 primary care medical ui ìits attending $36.5 \%$ of the population and approximately $50.9 \%$ of older adults. A total of 202 individuals from the 1252 involved in COSFOMA, agreed to participate in the study. In the COSFOMA study, participants were chosen according to their place of residence through a simple random selection from the list of older adults affiliated to IMSS from the 48 first-level clinics of Mexico City ${ }^{7}$. Written letters delivered to their home addresses were used as invitations to inform them of the nature of the study and to provide them with an appointment at their clinic. When the older adults did not attend the appointment. aphone invitation was made and in some cases, a home visit. Blood samples were obtained by venipunctureof the median cubital vein using the vacutainer system. Samples collected from April to September 2015 were used in this study.

\section{Ethics statement}

This research protocol was conducted with the approval of the National Committee of Scientific Research as well as by the Ethics Committee for Health 
Research of the Instituto Mexicano del Seguro Social (R-2012-785-067). All participants gave written informed consent. The study was performed according to the Code of Ethics of the World Medical Association Declaration of Helsinki.

\section{Evaluation of ROS}

To evaluate by fluorometry the formation of ROS, $5 \mu \mathrm{l}$ of serum and $85 \mu$ of PBS 1 X buffer were added to a $10 \mu$ solution of dichlorofluorescein (DCFH) diacetate (CAS Number 4091-99-0, Sigma-Aldrich), incubated in darkness for $30 \mathrm{~min}$ at $37^{\circ} \mathrm{C}$ to allow for oxidation of DCFH to the fluorescent compound 2-7-DCFH by the presence of hydrogen peroxide. Samples were then read at $498 \mathrm{~nm}$ excitation and $522 \mathrm{~nm}$ emission (Cytation 5 Cell Imaging Multi-Mode Reader), previously calibrated with a standard curve. Data are shown as $\mathrm{pmol} / \mu \mathrm{L}$ of serum.

\section{Determination of lipid peroxidation}

To evaluate lipid peroxidation, we measured malondialdehyde (MDA) as a reaction of thiobarbituric acid (CAS Number 504-17-6, Sigma-Aldrich); $50 \mu$ of serum with $25 \mu \mathrm{l}$ of PBS $1 \mathrm{X}$ were added to $50 \mu \mathrm{l}$ of thiobarbituric acid. The sample was placed in a boiling bath at $94^{\circ} \mathrm{C}$ for $20 \mathrm{~min}$, then centrifuged at 10,500 rpm for $15 \mathrm{~min}$; the supernatant was read at $532 \mathrm{~nm}$ with a spectrophotometer $(\mathrm{EPOCH})$, which was previously calibrated with a standard curve. Data are shown as $\mu \mathrm{mol} / \mu \mathrm{L}$ of serum.

\section{Sample processing and telomere length assessment}

Genomic DNA was extracted from peripheral leukocytes by the salting-out procedure. Purified DNA samples were aliquoted in a concentration of $10 \mathrm{ng} / \mu \mathrm{L}$ and stored at $-70^{\circ} \mathrm{C}$ until use. For telomere length assessment, we followed the quantitative polymerase chain reaction ( $q P C R$ ) method published by $\mathrm{O}^{\prime}$ Callaghan and Fenech20. The number of copies of telomere repeats was determined by the standard curve of Tel STD, while the standard curve of 36B4 STD was used as a housekeeping gene. After the GPCR reaction on a StepOnePlus real-time PCR System (Applied Biosystem), the $C t$ values of each sample were extrapolated in their corresponding curves by a linear regression test to determine telomere length. The Maxima SYBR
Green/ROX qPCR Master Mix 2X (Thermo $\$ Scientific, California, USA) was used. The cycling conditions for both genes were: $10 \mathrm{~min}$ at $95^{\circ} \mathrm{C}$, foillowed by 40 cycles of $95^{\circ} \mathrm{C}$ for $15 \mathrm{~s}, 60^{\circ} \mathrm{C}$ for 1 min, followed by a melting curve. Data are shown as kilobase-pairs (kb).

\section{Frailty}

The operationalization of the frailty phenotype was performed using the five criteria proposed by Fried et al. ${ }^{6}$ Frail adults are defined as those with three or more of the following criteria: self-report of wweight loss, exhaustion, low physical activity, slowness, and weakness (low grip strength). The presence of two criteria indicates a pre-frail condition, whereas the absence of criteria indicates a robust or non-frail state$^{7}$. Participants were classified as non-frait (score 0 ), pre-frail (score 1-2), and frail (score 3-5).

\section{Statistical analysis}

In general, the data analysis and visualizations were carried out with free (R) ${ }^{21}$ and commercial (SPSS and GraphPad) software. In particular, we used ggplot222 and ggsignif ${ }^{23} \mathrm{R}$ packages. Quantitative variabłes are presented as the arithmetic mean and stand $\overline{\text { arrd }}$ deviation (mean $\pm \mathrm{SD}$ ). Qualitative variables are presented as absolute ( $n$ ) and relative (\%) frequencies. Body mass index (BMI) was reclassified into nutritional status according to the WHO criteria (underweight $<18.5 \mathrm{~kg} / \mathrm{m}^{2}$, normal weight $18.5-24.9 \mathrm{~kg} / \mathrm{m}^{2}$ overweight $25-29.9 \mathrm{~kg} / \mathrm{m}^{2}$, and obesity $\geq 30 \mathrm{~kg} / \mathrm{m}^{2}$ ) to explore the possible correlation between tetomere length and nutritional status. To explore the distribution of DCFH (pmol/ $\mu \mathrm{L}$ of serum), MDA ( $\mu \mathrm{mol} / \mu \mathrm{L}$ of serum), and telomere length $(\mathrm{kb})$ between frailty categories (non-frail, pre-frail, and frail), we generated boxplots and calculated their p-value by MannWhitney Wilcoxon test with Bonferroni correction.

The relationship between DCFH and MDA with telomere length by frailty categories was explofed by scatter plots and linear regression with the Pearson correlation coefficient ( $r$ ). The distribution of telomere length and oxidative stress among oldep adult groups was evaluated calculating $p$-values obtained by Kruskal-Wallis non-parametric test. The ašsociation between three frailty categories with $\triangle \mathrm{DCFH}$, MDA, and telomere length was analyzed by 
Table 1. General characteristics, oxidative stress (reactive oxygen species and lipid peroxidation), and telomere length in a sample of Mexican older adults $60-95$ years of age $(n=202)$

\begin{tabular}{|c|c|c|}
\hline Characteristics & Participants & $n(\%)$ \\
\hline Age (mean \pm SD years) & $69.89 \pm 7.39$ & \\
\hline \multirow[t]{2}{*}{ Sex } & Female & $133(65.8)$ \\
\hline & Male & $69(34.2)$ \\
\hline BMI (mean \pm SD kg/m²) & $27.71 \pm 6.59$ & \\
\hline \multirow[t]{4}{*}{ Nutritional status } & Underweight & $8(3.96)$ \\
\hline & Normal weight & $63(31.19)$ \\
\hline & Overweight & $78(38.61)$ \\
\hline & Obesity & $53(26.24)$ \\
\hline \multirow[t]{3}{*}{ Years of school } & No schooling & $16(7.9)$ \\
\hline & $1-6$ years & $78(38.6)$ \\
\hline & $\geq 7$ years & $108(53.5)$ \\
\hline \multirow[t]{3}{*}{ Chronic illnesses } & None & $115(56.9)$ \\
\hline & 1 & $57(28.2)$ \\
\hline & $\geq 2$ & $30(14.9)$ \\
\hline \multirow[t]{2}{*}{ Smoking (currently) } & No & $187(92.6)$ \\
\hline & Yes & $15(7.4)$ \\
\hline \multirow[t]{2}{*}{ Drinking (currently) } & No & $168(83.2)$ \\
\hline & Yes & $34(16.8)$ \\
\hline \multirow[t]{3}{*}{ Frailty status } & Non-frail & $29(14.4)$ \\
\hline & Pre-frail & $122(60.4)$ \\
\hline & Frail & $51(25.2)$ \\
\hline $\begin{array}{l}\text { Reactive oxygen species (DCFH, } \\
\text { mean } \pm \mathrm{SD} \text { pmol } / \mu \mathrm{L} \text { ) }\end{array}$ & $50.27 \pm 24.58$ & \\
\hline $\begin{array}{l}\text { Lipid peroxidation (MDA, } \\
\text { mean } \pm \mathrm{SD} \mu \mathrm{mol} / \mu \mathrm{L} \text { ) }\end{array}$ & $33.53 \pm 13.18$ & \\
\hline Telomere length (mean $\pm \mathrm{kb}$ ) & $5.31 \pm 1.72$ & \\
\hline
\end{tabular}

DCFH: dichlorofluorescein; MDA: malondialdehyde; SD: standard deviation.

multinomial logistic regression analysis with $\mathrm{p}$-values obtained by Student's t-test. Previous analyses were carried out to determine a possible linear correlation between oxidative stress, lipid peroxidation, and telomere length in three conditions: non-frail, pre-frail, and frail groups of older adults. However, to establish the magnitude of the association against the frailty category, we performed a logistic regression considering the non-frail group as the reference category to compare the odds of being classified as pre-frail or frail considering oxidative stress (DCFH or MDA) or telomere length. The odds ratio (ORs) with $95 \%$ confidence intervals $(95 \% \mathrm{Cl})$ was obtained for precision. Pearson correlation coefficients, as well as multinomial logistic regression analysis, were âdjusted for age, BMI, chronic illnesses $(0,1, \geq 2)$, educational level (without studies, $1-6$ years, and $\geq 7 \bar{y}$ ears), drinking (binary), smoking (binary), and sex (binary), as possible confounders.

\section{RESULTS}

From the random selection of participants in the COSFOMA study, we invited 1252 members and recruited 202 older adults ( $\geq 60$ years of age) who agreed to participate in this study. Table 1 shows the general characteristics as well as ROS measured by DCFH, 
Figure 1. Oxidative stress and telomere length among older adults. The figure shows the distribution of dichlorofluorescein $(p=0.76)$, malondialdehyde $(p=0.37)$, and telomere length $(p<0.001)$, among frailty groups (green: non-frail; yellow: prefrail, and red: frail). The $p$-value was obtained by Kruskal-Wallis nonparametric test. Panels A, B, and C show with thick lines the differences within frail categories while panels $\mathbf{D}, \mathbf{E}$, and $\mathbf{F}$ show the differences between females and males by MannWhitney Wilcoxon test. Bonferroni correction $<0.016$ was calculated to assign significant differences. ${ }^{* * *} p$-values $<0.001$. p-value was obtained by Kruskal-Wallis non-parametric test.
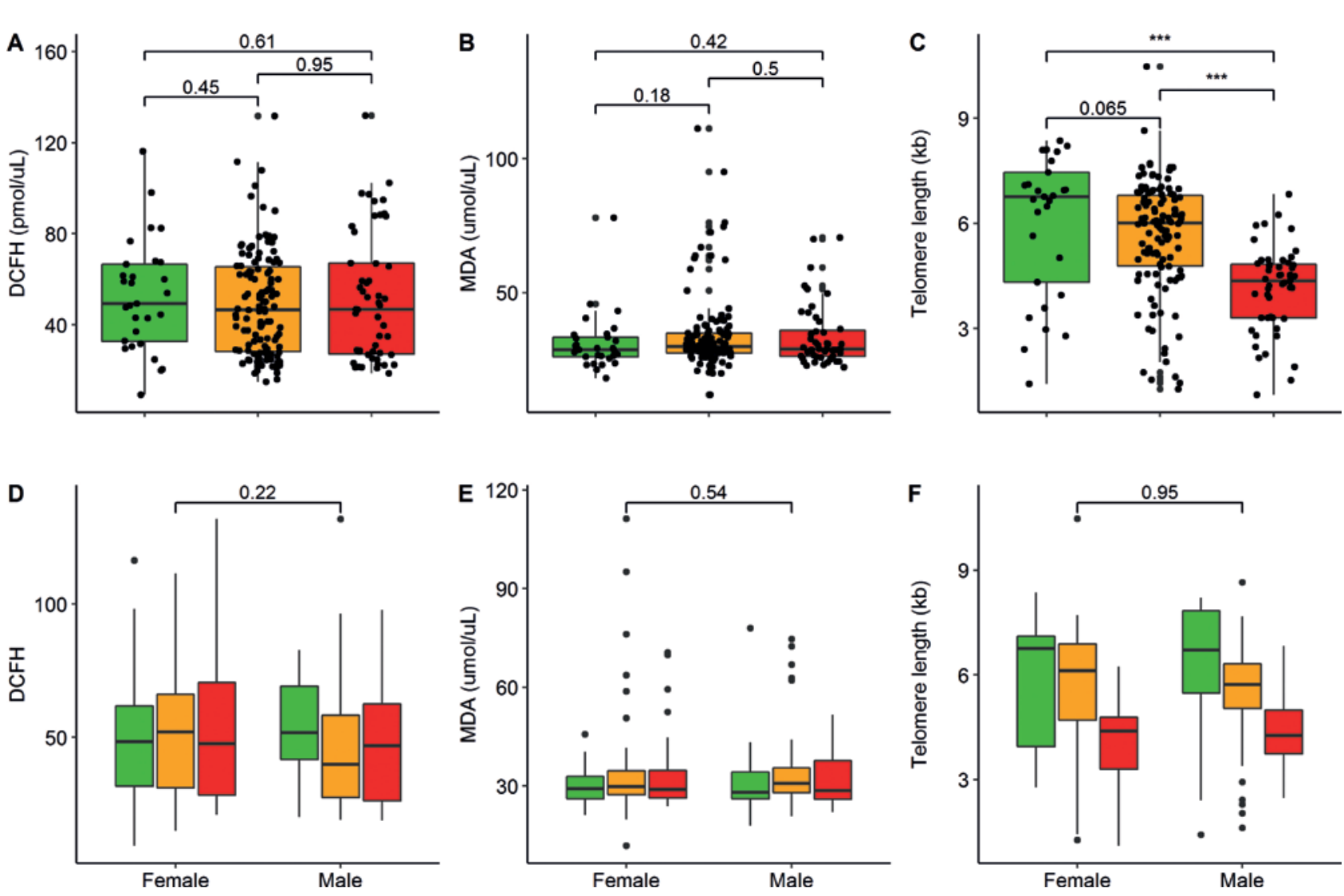

lipid peroxidation measured by MDA, and telomere length measurements of the older adults, $133 \mathrm{fe}$ males (65.8\%) and 69 males (34.2\%), ranging between 60 and 95 years of age (mean age: $68.9 \pm 7.4$ ). Regarding the frailty status, $14.4 \%$ of participants were classified as non-frail, $60.4 \%$ as pre-frail, and $25.2 \%$ as frail.

The aim of this study was to determine a possible association between oxidative stress measured by ROS (DCFH) and lipid peroxidation (MDA), telomere length, and frailty status. We hypothesized that older adults with frailty would have increased oxidative stress measurements associated with shorter telomeres when compared with both pre-frail and nonfrail subjects. Contrary to our hypothesis, we observed no significant differences between ROS (DCFH, $p=0.76)$ and lipid peroxidation (MDA, $p=0.37$ ) among the frailty categories, whether dividedor not by sex. Between frailty categories, we did find a significant difference in telomere length ( $p<$ 0.001). This difference was explained by lower tefomere length in the frail group compared with the nôn-frail $(p<0.001)$ and the pre-frail $(p<0.001)$ groups, while the differences between non-frail and pre-frati were not statistically significant $(p=0.065)$ (Fig. 1).

Then, to assess the possible association between lipid peroxidation (MDA), ROS (DCFH), and tefomere length with groups of older adults, we performed a multinomial logistic regression adjusted by age, sex, BMI, chronic illnesses, educational level, drinking, and smoking. Figure 2 shows the magnitude and precision of the association of oxidative stress and telomere 
Figure 2. Association of oxidative stress and telomere length in Mexican older adults. The forest plot shows the multinomial logistic regression considering the non-frail group as the reference category to calculate the odds of being classified as pre-frail or frail considering oxidative stress (dichlorofluorescein or malondialdehyde) or telomere length. The odds ratio with $9 \mathbf{5} \%$ confidence interval was obtained for precision and adjusted by age, sex, body mass index, educational level, chronic illnesses, smoking, and drinking. Bold text indicates a statistically significant difference ( $p$-value cutoff $<0.05$ ).

\section{$\triangle \mathrm{DCFH}(\mathrm{pmol} / \mathrm{uL})$ \\ MDA (umol/uL) \\ Telomere $(\mathrm{kb})$
$\nabla \mathrm{DCFH}(\mathrm{pmol} / \mathrm{uL})$}

MDA (umol/uL)

Telomere (kb)

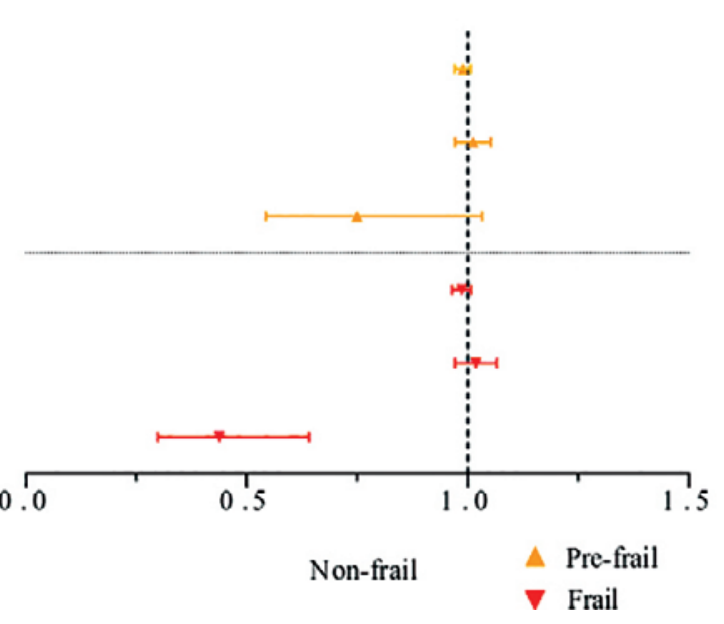

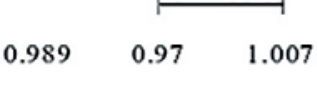

$1.011 \quad 0.971 \quad 1.052$

$0.749 \quad 0.543 \quad 1.032$

$0.986 \quad 0.964 \quad 1.008$

$1.018 \quad 0.971 \quad 1.066$

0.438

0.299

0.641
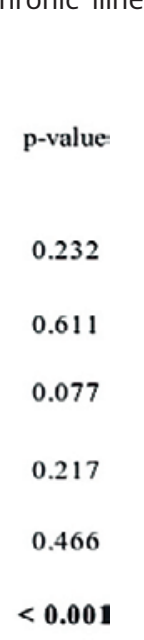

length in pre-frail and frail older adults when considering the non-frail subjects as the reference category. We observed that the odds of being classified in the pre-frail group increased 0.989 per each pmol of DCFH (95\% Cl [0.97-1.007]; $p=0.232$ ), and 1.011 per each $\mu \mathrm{mol}$ of MDA (95\% Cl [0.971-1.052]; $p=0.611$ ), while every $\mathrm{kb}$ of telomere (a long telomere) protected 1.33 times from a pre-frail classification $(\mathrm{OR}=0.749,95 \% \mathrm{Cl}[0.543-1.032] ; \mathrm{p}=0.012)$. On the other hand, the odds of being classified in the frail group increased 0.986 per each pmol of DCFH (95\% Cl [0.964-1.008]; $p=0.217)$, and 1.018 per each $\mu \mathrm{mol}$ of MDA (95\%Cl [0.971-1.066]; $p=0.466$ ), while every kb of telomere (a longer telomere) protected 2.28 times from a frailty classification $(\mathrm{OR}=$ $0.438,95 \% \mathrm{Cl}$ [0.299-0.641]; $\mathrm{p}<0.001)$.

Finally, we evaluated the relationship between DCFH and MDA on telomere length according to frailty status. First, we calculated the global effect of both DCFH $(r=-0.031, p=0.66)$ and MDA $(r=0.095, p=0.18)$ on telomere length and observed no significant correlation between ROS or lipid peroxidation and telomere length. Then, we performed the analysis for each frailty category (Fig. 3). In addition, since the effect of obesity or adiposity on telomere length has been reported previously 24,25 , and although a negative association of BMI with telomere length is reduced among older adults ${ }^{25}$, we explored the possible correlation between telomere length and nutritional status (underweight, normal weight, overweight, and obesity), no significant association was found with any of the groups: underweight $(r=0.640, p=0.088)$, normal weight $(r=0.089, p=0.49)$, overweight $(r=$ $0.110, p=0.33)$, and obesity $(r=0.027, p=0.85)$, nor when subjects were divided by frailty categories (data not shown).

\section{DISCUSSION}

Frailty, telomere length, and oxidative stress were evaluated in a group of older adults to define their association and correlation. A first analysis wầs conducted to determine our hypothesized distinct distributions between oxidative stress, lipid péroxidation, and telomere length according to frailty groups; however, we observed a significantly different2distribution only for telomere length. Telomere length was found to be associated with pre-frail and frail categories, as previously reported ${ }^{12}$. This specifit variable has become of importance for older a dults since, despite the philosophical debate about the definition of frailty, it has shown to have a predictive 
Figure 3. Relationship between oxidative stress and telomere length among frailty categories. The figure shows the correlation analysis for A: dichlorofluorescein and B: malondialdehyde, with telomere length. The analysis was conducted on thevoverall study population. Linear regression for non-frail (green; square), pre-frail (yellow; triangle), and frail (red; inverted triangle) participants with Pearson's correlation coefficients $(r)$ and $p$-values adjusted by age, sex, body mass index, educational level, chronic illnesses, smoking, and drinking is shown.

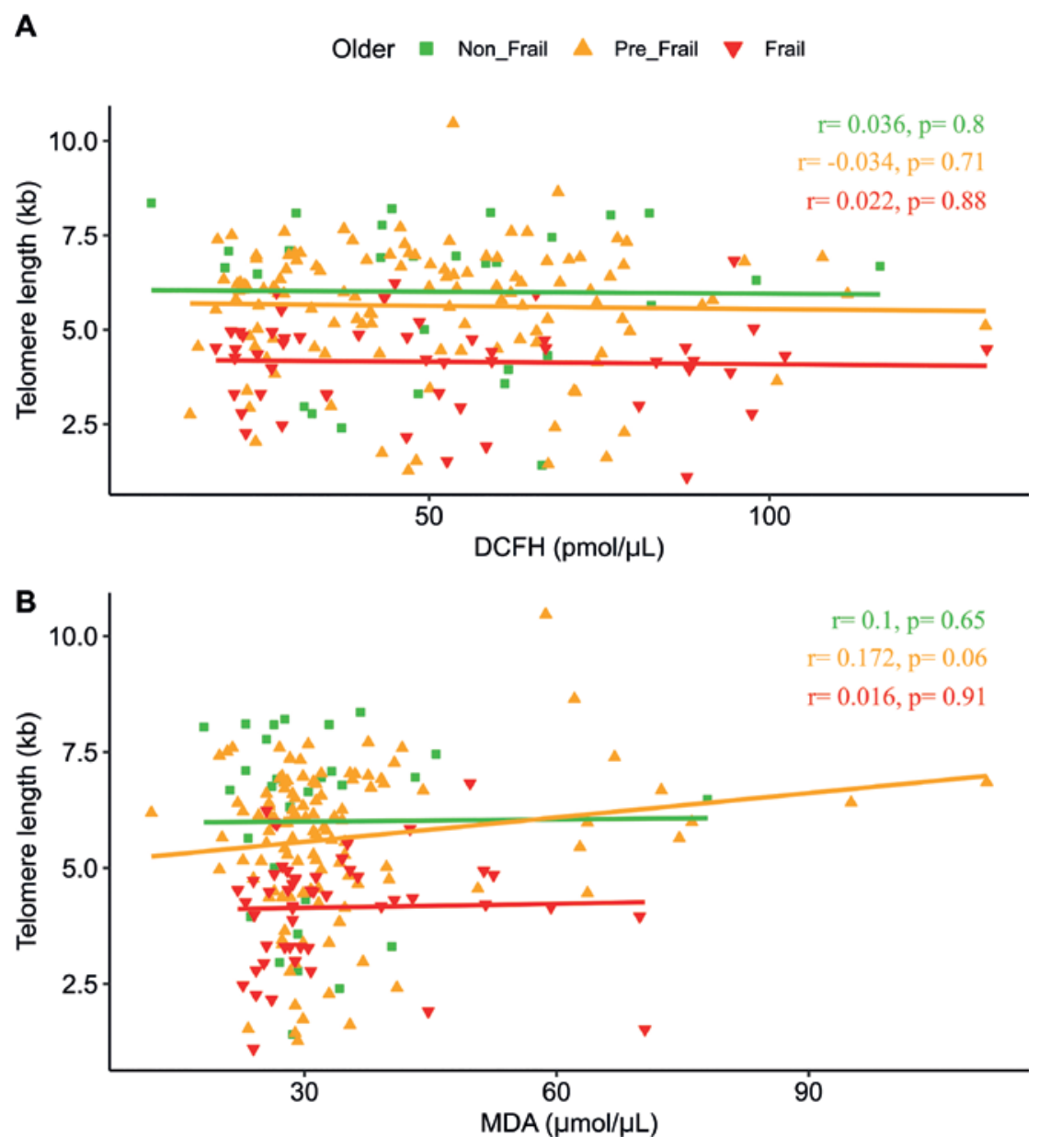

value on poor health outcomes. Here, we have found that a shorter telomere, which by itself has been previously related to a briefer lifespan, is associated with the frailty status. Even more, when considering the telomere length of non-frail older adults as the reference category, short telomeres were associated with pre-frail older adults while even shorter telomere length was associated with the frail status. As these results were adjusted for age, sex, BMI, chronic illnesses, educational level, drinking, and smoking, possible confounders included in our analysis, we consider that telomere length is a strong candidate as a biomarker of frailty status. To confirm this, the present study must be replicated in other populations for telomere length to be considered as a biomarker of frailty since telomere length is affected by environmental factors ${ }^{26}$.

Telomere length is associated with modifiable factors such as smoking and obesity, which are related to shorter telomeres, while exercise and a healtiby diet preserve telomere length ${ }^{4,27}$. Furthermore, astudy showed that frailty can be modified by ex ercise and nutrition, reversing the frailty state to non $u$ frail ${ }^{28}$. Hence, telomere length determination as part of a frailty diagnosis could help in the prompt implementation of intervention measures, in either the pre-frail or frail status, on the above-mentioned factors to reverse this condition. If pre-frail status and frailty are assessed on time, more costly medical outcomes 
could be prevented, and quality of life improved. Research in this direction may help determine frailty in a more quantitative manner with a biological background. Although telomere length determination currently is not widely available, its future implementation could be considered as part of the frailty diagnosis, as a non-invasive tool. On that matter, the association of frailty status with telomere length could mean that besides its definition, frailty has a biological counterpart that changes according to the frailty status. Hence, we evaluated oxidative stress as a probable cause of telomere shortening that could be assessed as telomere length and found a mild effect of MDA on telomere length $(r=0.17 ; p=0.06)$ for the pre-frail category. MDA is a marker for lipid peroxidation, i.e., the oxidative degradation of lipids that follows the excess of free radical formation. The increase of MDA in pre-frail subjects and the decrease in telomere length between pre-frail and frail subjects suggest that oxidative stress affects telomere length regarding a frailty status in older adults.

The main limitation of the present study was that even when the original cohort employed consisted of 1252 individuals, we could only include 202 participants, meaning our recruitment skills were insufficient. The hypothesis generated in this study could be explored in a longitudinal study that would provide further information on the effect of MDA on telomere length and frailty status. Furthermore, a more thorough analysis of oxidative stress, specifically the measurement of 8-hydroxy-2'-deoxyguanosine, a specific biomarker of oxidative stress in DNA ${ }^{29}$, would enrich the knowledge regarding the effect of these two biological outcomes of frailty. It is worth mentioning that when drinking, smoking, and chronic illnesses were not considered as confounder variables, we observed a linear association between MDA and telomere length $(p=0.089)$. We are convinced that an ideal approach would have these as exclusion criteria to avoid masking this biological measurement.

We confirmed an association of telomere length with the frailty phenotype and found a low association between lipid peroxidation and telomere length. However, we were unable to prove or disprove whether the cause of telomere length changes in frail patients is due to oxidative stress. Moreover, we found no effect of drinking or smoking as confounder variables regarding telomere length or oxidative stress older adults in this study.

\section{Availability of data and material}

The datasets analyzed in the present stuady are available at the Open Science Framework repository link: https://osf.io/apx35/?view_only=40fca3044c 7a4ca7a1b5fba62185d41f.

\section{ACKNOWLEDGMENTS}

This study was supported by CONACYT (Consejo Nacional de Ciencia y Tecnología, Mexico) grant SALUD-2013-01-201112. The authors thankMario Enrique Rendón-Macías for his comments on data analysis and visualization. This study was performed in partial fulfillment of the requirements for the PhD degree of JDME, who thanks the Posgrado en Ciencias Biológicas, Biología Experimental of the National University of Mexico (UNAM), Mexico.

\section{REFERENCES}

1. WHO. 10 Facts on Ageing and Health; 2017. Available from: http://www.who.int/features/factfiles/ageing/en. [last accessed on 2018 Mar 05].

2. González KD. Envejecimiento Demográfico en México:-análisis Comparativo Entre Las Entidades Federativas. Consejo Nacional de Población CONAPO. Consejo Nacional de Poblaciōn; 2016. Available from: http://www.conapo.gob.mx/en/CONAPO/Envejecimiento_demografico_en_Mexico. [Last accessedon 2018 Mar 05].

3. Análisis Prospectivo de la Población de 60 Años de Edad en Adelante. Secretaría de Desarrollo Social; 2017. https:/ /www. gob.mx/cms/uploads/attachment/file/201801/An_lisis_prospectivo de la poblaci n de 60_a_os_en_adelante.pdf.

4. Rowe $J \bar{W}, \overline{K a h n}$ RL. Successful aging. Gerontologist. 1997; 37:433-40.

5. Buckinx F, Rolland Y, Reginster JY, Ricour C, Petermans $]$, Bruyère $O$, et al. Burden of frailty in the elderly population: perspectives for a public health challenge. Arch Public Health. 2015;73:19.

6. Fried LP, Tangen CM, Walston J, Newman AB, Hirsch Cottdiener J, et al. Frailty in older adults: evidence for a phenotype. J Gerontol A Biol Sci Med Sci. 2001;56:M146-56.

7. Sánchez-García S, García-Peña C, Salvà A, Sánchez-Arenas R, Granados-García V, Cuadros-Moreno J, et al. Frailty incommunity-dwelling older adults: association with adverse outcomes. Clin Interv Aging. 2017;12:1003-11.

8. Collard RM, Boter H, Schoevers RA, Oude Voshaar RC. Prevalence of frailty in community-dwelling older persons: acsystematic review. J Am Geriatr Soc. 2012;60:1487-92.

9. Boonekamp JJ, Simons MJ, Hemerik L, Verhulst S. Telomere length behaves as biomarker of somatic redundancy rather than biological age. Aging Cell. 2013;12:330-2.

10. Woo J, Yu R, Tang N, Leung J. Telomere length is associated with decline in grip strength in older persons aged 65 years and over. Age (Dordr). 2014;36:9711. 
11. Breitling LP, Saum KU, Perna L, Schöttker B, Holleczek B, Brenner $\mathrm{H}$, et al. Frailty is associated with the epigenetic clock but not with telomere length in a German cohort. Clin Epigenetics. 2016;8:21.

12. Ortiz-Ramírez M, Sánchez-García S, García-Dela Torre P, ReyesMaldonado E, Sánchez-Arenas R, Rosas-Vargas H, et al. Telomere shortening and frailty in Mexican older adults. Geriatr Gerontol Int. 2018;18:1286-92.

13. Zee RY, Castonguay AJ, Barton NS, Germer S, Martin M. Mean leukocyte telomere length shortening and Type 2 diabetes mellitus: a case-control study. Transl Res. 2010;155:166-9.

14. Nordfjäll K, Eliasson M, Stegmayr B, Lundin S, Roos G, Nilsson $\mathrm{PM}$, et al. Increased abdominal obesity, adverse psychosocial factors and shorter telomere length in subjects reporting early ageing; the MONICA northern Sweden study. Scand J Public Health. 2008;36:744-52

15. D'Mello MJ, Ross SA, Briel M, Anand SS, Gerstein H, Paré G, et al. Association between shortened leukocyte telomere length and cardiometabolic outcomes: systematic review and metaanalysis. Circ Cardiovasc Genet. 2015;8:82-90.

16. Pan $H Z$, Zhang $H$, Chang $D$, Li H, Sui $H$. The change of oxidative stress products in diabetes mellitus and diabetic retinopathy. $\mathrm{Br}$ J Ophthalmol. 2008;92:548-51.

17. Safar ME, O'Rourke M, Frohlich ED, editors. Blood Pressure and Arterial Wall Mechanics in Cardiovascular Diseases. London: Springer; 2014. p. 567

18. Rodríguez-Castañeda A, Martínez-González KL, Sánchez-Arenas R, Sánchez-García S, Grijalva I, Basurto-Acevedo L, et al. Oxidative stress in the elderly with diabetes mellitus or hypertension Rev Med Inst Mex Seguro Soc. 2018;56:S12-7.
19. Ahmed $W$, Lingner J. Impact of oxidative stress on felomere biology. Differentiation. 2018;99:21-7

20. O'Callaghan NJ, Fenech M. A quantitative PCR methodfor measuring absolute telomere length. Biol Proced Online. 2011;13:3.

21. R: The R Project for Statistical Computing. Avaliable from: https://www.r-project.org. Last accessed on 2018 Aug 08].

22. Wickham H. ggplot2. New York: Springer; 2009

23. Ahlmann-Eltze C. Significance Brackets for "ggplot2" [R Package Ggsignif Version 0.4.0]. Available from: https://www. CRAN.R-project.org/package=ggsignif. [Last accessed on 2018 Aug 08].

24. Mundstock E, Sarria EE, Zatti H, Mattos Louzada F, Kiç Grun L, Herbert Jones $M$, et al. Effect of obesity on telomere length: systematic review and meta-analysis. Obesity (Silverspring). 2015;23:2165-74.

25. Lee M, Martin H, Firpo MA, Demerath EW. Inverse association between adiposity and telomere length: the fels longitudinal study. Am J Hum Biol. 2011;23:100-6.

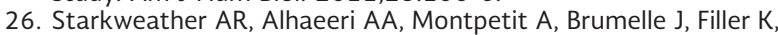
Montpetit $M$, et al. An integrative review of factors associated with telomere length and implications for biobehavioral research. Nurs Res. 2014;63:36-50.

27. Shammas MA. Telomeres, lifestyle, cancer, and aging. Eurr Opin Clin Nutr Metab Care. 2011;14:28-34

28. Michel JP, Cruz-Jentoft AJ, Cederholm T. Frailty, exercise and nutrition. Clin Geriatr Med. 2015;31:375-87.

29. Te Koppele JM, Lucassen PJ, Sakkee AN, Van Asten JG, Ravid R, Swaab DF, et al. $80 \mathrm{HdG}$ levels in brain do not indicate oxidative DNA damage in alzheimer's disease. Neurobiol Aging. 1996; 17:819-26 